Fábio José Buainain Barcellos de Paula

\title{
A gestão do conhecimento como estratégia competitiva em situações de fusão e aquisição - O caso Xerox|MHW
}

Dissertação de MEstrado

DePARTAMENTO de AdMinistraçÃo Programa de Pós-graduação em Administração de Empresas 


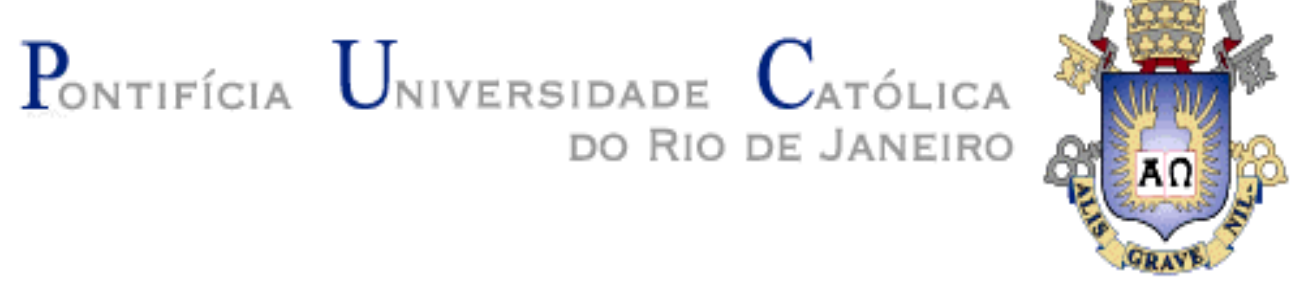

Fábio José Buainain Barcellos de Paula

A gestão do conhecimento como estratégia competitiva em situações de fusão e aquisição - O caso Xerox|MHW

\begin{abstract}
Dissertação de Mestrado
Dissertação apresentada ao Departamento de Administração da PUC-Rio - Programa de Pósgraduação em Administração de Empresas, como parte dos requisitos para obtenção do título de Mestre em Administração de Empresas.
\end{abstract}

Orientadora: Profa. T. Diana L. v. A. de Macedo-Soares

Rio de janeiro, 05 de Setembro de 2006 


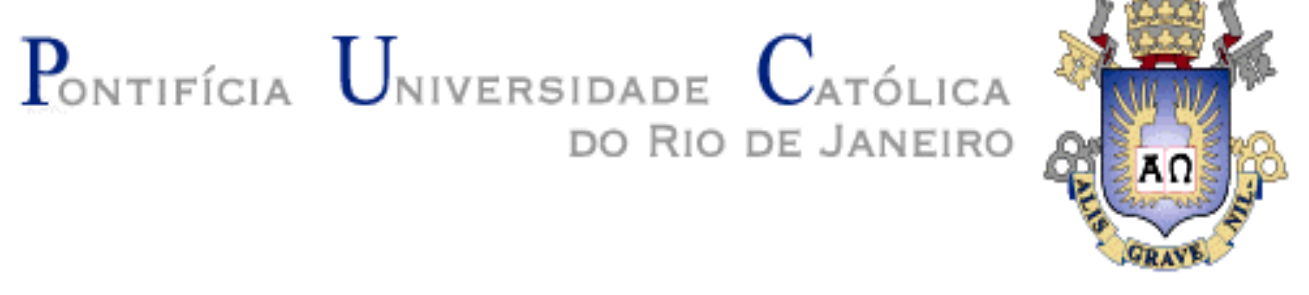

Fábio José Buainain Barcellos de Paula

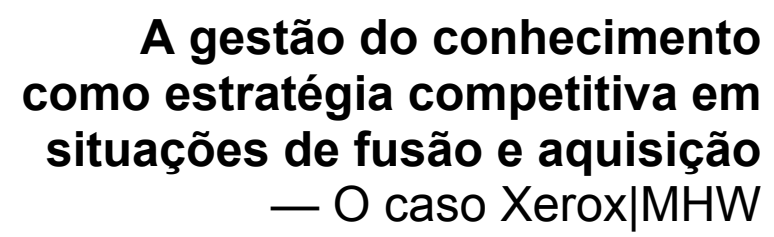

Dissertação apresentada ao Departamento de Administração da PUC-Rio - Programa de Pósgraduação em Administração de Empresas, como parte dos requisitos para obtenção do título de Mestre em Administração de Empresas. Aprovada pela comissão examinadora abaixo assinada.

Profa. T. Diana L. v. A. de Macedo-Soares Orientadora

Departamento de Administração - PUC-Rio

Prof. José Roberto Gomes da Silva Departamento de Administração - PUC-Rio

Profa. Adriane Barbosa Cavalieri INT - Instituto Nacional de Tecnologia

Prof. João Pontes Nogueira Vice-Decano de Pós-Graduação do CCS

Rio de janeiro, 05 de setembro de 2006 
Todos os direitos reservados. É proibida a reprodução total ou parcial do trabalho sem autorização da PUC-Rio, do autor e da orientadora.

Fábio José Buainain Barcellos de Paula

Graduado em Economia pela Universidade Federal do Rio de Janeiro, em 1997. Sócio-Diretor da empresa QuickMind Knowledge Management e ex-sócio da empresa MHW. Coautor do estudo Estratégia para o mercado de e-learning no Brasil: o caso Xerox|MHW, junto com T. Diana L. v. A. de Macedo-Soares.

Ficha Catalográfica

Paula, Fábio José Buainain Barcellos de

A gestão do conhecimento como estratégia competitiva em situações de fusão e aquisição - o caso Xerox/MHW / Fábio José Buainain Barcellos de Paula ; orientadora: T. Diana L. V. A. de Macedo-Soares. 2006.

292 f. : il. ; $30 \mathrm{~cm}$

Dissertação (Mestrado em Administração)Pontifícia Universidade Católica do Rio de Janeiro, Rio de Janeiro, 2006.

Inclui bibliografia

1. Administração - Teses. 2. Estratégia competitiva. 3. Competências organizacionais. II. 4. Gestão do conhecimento. 5. Fusão e aquisição. 6 . Adequação estratégica. I. Macedo-Soares, T. Diana L. v. A. de. II. Pontifícia Universidade Católica do Rio de Janeiro. Departamento de Administração. III. Título.

CDD: 658 


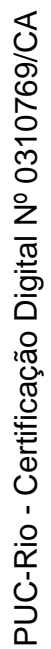

Para minha família, pelo apoio e confiança incondicionais ao longo dessa jornada. 


\section{Agradecimentos}

Concluir com sucesso mais esse ciclo da minha vida não teria sido possível sem a contribuição e apoio de familiares, amigos, professores, funcionários, exfuncionários, sócios, ex-sócios... Mas algumas pessoas foram especialmente decisivas para que eu lograsse atingir esse objetivo e, por isso, não poderia deixar de agradecê-las nominalmente.

À minha avó Regina Vera, pelo patrocínio financeiro concedido para pagar boa parte do meu curso de mestrado.

Ao meu pai Jardel, que esteve incansavelmente ao meu lado, incentivando, cobrando resultados, indicando novas bibliografias, revisando inúmeras vezes o texto, dando importantes feedbacks e idéias que melhoraram em muito a pesquisa.

À minha professora orientadora, Diana Macedo-Soares, pela contribuição, paciência e por acreditar em mim até o final.

Aos professores que participaram da Comissão examinadora, José Roberto Gomes da Silva e Adriane Barbosa Cavalieri.

Ao professor Jorge Ferreira que aceitou meus sucessivos pedidos de extensão de prazo para que eu finalizasse o trabalho.

Aos solícitos funcionários da Secretaria Teresa, Fabio e Leopoldo pela ajuda ao longo de todos esses anos.

E à minha filha Isabella, que soube esperar, pacientemente, que o papai defendesse a dissertação no dia 05 de setembro, deixando para nascer no dia seguinte. 


\section{Resumo}

Barcellos-de-Paula, Fabio José B..; Macedo-Soares, T. Diana L. v. A. A Gestão do Conhecimento em Situações de Fusão e Aquisição - O Caso da Xerox|MHW. Rio de Janeiro, 2006. 292p. Dissertação de Mestrado - Departamento de Administração, Pontifícia Universidade Católica do Rio de Janeiro.

O conhecimento organizacional, ou capital intelectual, é hoje reconhecido como o fator fundamental não só para o crescimento, como para a própria sobrevivência das organizações em ambientes dinâmicos, turbulentos, incertos, altamente inovadores e competitivos, como os do presente contexto de economias crescentemente globalizadas. A gestão do conhecimento organizacional (KM) apresenta-se, então, como uma das condições necessárias ao sucesso de estratégias competitivas, embora, por certo, não suficiente, sendo a demanda de conhecimento externo existente, por meio de negócios de fusão e aquisição (M\&A), reconhecida como um dos processos essenciais da gestão, alternativamente a procedimentos inovadores em P\&D, em geral lentos e onerosos. Todavia, pesquisas têm comprovado que, mundialmente, apenas cerca de $30 \%$ dos casos de M\&A têm alcançado sucesso em seus objetivos estratégicos. Não obstante, esse tipo de negócio tem se afigurado como essencial à expansão de empresas de classe mundial, dada a insuficiência do mero crescimento orgânico, o que constitui um paradoxo. Este estudo de caso teve por objetivo descrever e explicar as causas do fenômeno do insucesso no desempenho da estratégia da empresa Xerox|MHW, combinada em processo de aquisição, tendo como componente essencial a gestão do conhecimento. A literatura examinada para a compreensão dos conceitos básicos pertinentes contemplou tópicos sobre padrões organizacionais; estratégias de organizações; conhecimento organizacional e sua gestão e resultados de processos de fusão e aquisição de empresas. A coleta de evidências, adotando procedimentos de triangulação, foi realizada a partir de documentos, entrevistas e duas pesquisas, visando, a segunda, validar os dados coletados anteriormente. Com base no modelo integrativo-sistêmico GI de MACEDO-SOARES (2000), a realidade revelada pela pesquisa mostrou-se contraditória relativamente aos requisitos, pressupostos e suposições implícitos na formulação estratégica, resultando, daí, a sua não-adequação ao desempenho da organização na busca de seu objetivo estratégico. A conclusão é que um processo de aquisição está longe de ser algo 
trivial, sendo os problemas de não-integração organizacional, principalmente cultural, em casos de aquisição por escopo, em particular envolvendo uma grande empresa burocrática e uma pequena empresa de padrão organizacional flexível, os grandes responsáveis por esse elevado percentual. Quanto ao conhecimento organizacional, evidenciou-se que não basta a uma organização entendê-lo como um recurso essencial no contexto de negócios atual. É imprescindível a implementação de procedimentos e práticas para a sua gestão, não obstante constituir processo recente e complexo que requer, até por isso mesmo, efetivo apoio da alta administração das organizações e o desenvolvimento de uma cultura adequada à sua efetivação.

\section{Palavras-chave}

Estratégia competitiva; competências organizacionais; gestão do conhecimento; fusão e aquisição; adequação estratégica. 


\section{Abstract}

Barcellos-de-Paula, Fabio José B..; Macedo-Soares, T. Diana L. v. A. A Gestão do Conhecimento em Situações de Fusão e Aquisição - 0 Caso da Xerox|MHW. Rio de Janeiro, 2006. 292p. Dissertação de Mestrado - Departamento de Administração, Pontifícia Universidade Católica do Rio de Janeiro.

Organizational knowledge, or intellectual capital, is nowadays recognized as a key factor not only for growth, but for survival of organizations in dynamic, disturbed, uncertain and highly innovative and competitive environments, such as the ones in the current scenario of rising global economies. Organizational knowledge management (KM) is introduced, therefore, as one of the necessary conditions for competitive strategies success. However, of course, it is not enough, and the need for external knowledge, through merger and acquisition (M\&A), is recognized as an essential management process, an alternative for innovative P\&D procedure, normally slow and burdensome. Anyway, researches have assured that, worldwide, only $30 \%$ of M\&A cases have reached success in their business goals. Nevertheless, this kind of business have been considered essential for the expansion of worldwide companies, since mere organic growth is insufficient, which is a paradox. This case study aims to describe and explain the causes for the failure phenomena in Xerox|MHW company strategy performance, combined when Xerox acquired MHW, with knowledge management as its key component. The literature consulted to comprehend these basic concerning concepts encompasses topics about organizational models, strategies, knowledge management and results of company merger and acquisition processes. The gathering of evidences, adopting triangulation procedures, has been performed using documents, interviews and two surveys. The second survey aimed to validate the data collected previously. Based on MACEDO-SOARES (2000) GI systemic-integrative model, the reality revealed by the research is contradictory in relation to the requirements, presuppositions and suppositions implicit in the strategy conception, resulting, from this, its nonadjustment to the organization performance, trying to pursuit its strategic objective. 
The conclusion is that an acquisition process is far from being something trivial, and the problems of organizational non-adjustment - mainly cultural, in cases of scope acquisition, particularly involving a major bureaucratic company and a minor one with flexible organization model - are the responsible factors for this high percentage. About organizational knowledge, it is clear that understanding it as an essential resource in the current business scenario is not enough. It is crucial the implementation of practices and procedures to its management. However, building this new and complex process requires, because of it, solid support from high level management in the companies and the development of a proper culture, fitting its accomplishment.

\section{Keywords}

Competitive strategy; organizational competencies; knowledge management; merger and acquisition; strategic fit. 


\section{Sumário}

1 INTRODUÇÃO 19

1.1. O problema 19

1.1.1. Formulação do problema 26

1.2. Objetivo da pesquisa 27

1.2.1. Questões intermediárias 28

1.3. Relevância do estudo $\quad 29$

1.4. Delimitação do estudo 30

1.5. Estrutura do documento 31

2 REFERENCIAL TEÓRICO 33

2.1. Revisão da literatura 33

2.1.1. Padrões organizacionais $\quad 34$

2.1.2. Cultura organizacional e sua dinâmica 47

2.1.3. Política e poder nas organizações $\quad 51$

2.1.4. Aprendizagem organizacional e mudança 53

2.1.5. Estratégias organizacionais 62

2.1.6. Gestão do conhecimento - fator essencial da estratégia $\begin{array}{ll}\text { competitiva } & 79\end{array}$

2.1.7. Combinações estratégicas organizacionais 119

2.2. Conceitos centrais de referência 135

2.3. O modelo integrativo-sistêmico GI de análise da adequação

estratégica 144

2.4. Roteiro para análise da adequação estratégica da organização

$\begin{array}{ll}\text { combinada } & 155\end{array}$

3 METODOLOGIA 156

3.1. Delineamento da pesquisa quanto ao método de coleta de dados 156

3.1.1. Caracterização quanto aos fins da pesquisa 158

3.1.2. Caracterização da pesquisa quanto aos meios utilizados 158

3.2. Etapas do processo da pesquisa 160

3.3. Unidade da pesquisa 161

3.4. Seleção dos sujeitos da pesquisa 162 
3.5. Coleta de dados 162

3.5.1. Os questionários da pesquisa 163

3.5.2. As entrevistas 164

3.5.3. Material de revisão da literatura 164

3.6. Tratamento dos dados 165

3.7. Limitações da pesquisa 166

4 RESULTADOS DA PESQUISA E SUA INTERPRETAÇÃO 168

4.1. Contextualização do fenômeno em estudo 169

4.1.1. As oportunidades no campo da educação e o mercado de e$\begin{array}{ll}\text { learning } & 169\end{array}$

4.1.2. Aspectos históricos das empresas objeto do negócio de M\&A 172

4.1.3. Caracterização dos perfis organizacionais da USB Xerox e da MHW

4.1.4. Caracterização da estratégia formulada para a empresa Xerox|MHW

4.2. Implicações dos fatores organizacionais, inclusive da KM, no desempenho da estratégia da empresa combinada Xerox|MHW

4.2.1. Quadro analítico consolidado dos resultados da pesquisa a partir do questionário semi-estruturado $B$ e com base no modelo $G /$ de Macedo-Soares

4.2.2. Análise e interpretação dos resultados da pesquisa relacionada aos fatores organizacionais da empresa combinada Xerox|MHW

4.3. Implicações dos fatores macroambientais e dos atores estratégicos no desempenho da empresa combinada Xerox|MHW

4.4. Apuração dos indicadores de desempenho da empresa combinada Xerox|MHW e avaliação da adequação da estratégia

\section{DISCUSSÃO DO FENÔMENO}

5.1. O processo de M\&A

5.1.1. Operacionalização das principais variáveis do fenômeno e suas relações

5.2. A não-adequação da estratégia formulada para a empresa combinada Xerox|MHW

5.2.1. Inconsistências relacionadas aos fatores organizacionais 246

5.2.2. Fatores que mais contribuíram para o insucesso da estratégia 247

5.2.3. Ameaças dos fatores macroambientais e dos atores estratégicos $\quad 249$ 
5.3. Fatores que teriam contribuído para o sucesso da estratégia 250

5.4. Avaliação da pesquisa e consolidação dos resultados 251

5.4.1. A pesquisa e suas limitações 252

5.4.2. Querer versus poder (fazer) 253

6 CONCLUSÕES E RECOMENDAÇÕES 257

6.1. Recomendações e sugestões 263

7 REFERÊNCIAS BIBLIOGRÁFICAS 264

8 ANEXOS 273

8.1. QUESTIONÁRIO SEMI-ESTRUTURADO A DA PESQUISA 274

8.2. QUESTIONÁRIO SEMI-ESTRUTURADO B DA PESQUISA 282 


\section{Lista de Figuras}

Figura 1 - A organização vista como um conjunto interdependente de subsistemas (com base em Morgan, 1996:52).

Figura 2 - Perfil das características organizacionais, com base em Morgan (1996:66).

Figura 3 - Diagrama de fluxos do processo de aprendizagem por circuito simples e por circuito duplo (Argyris \& Schön, 1978).

Figura 4 - Diagrama dos componentes da base de conhecimento e relações entre níveis na hierarquia conceitual do conhecimento, com fundamento em Probst et al. (2002).

Figura 5 - Capital intelectual segundo Saint-Onge (Stewart, 1998:228).

Figura 6 - Capital intelectual segundo Edvinsson (Stewart, 1998:228).

Figura 7 - Capital intelectual (capital do conhecimento) com base em Bukowitz \& Williams (2002).

Figura 8 - Gestão do conhecimento com base em Bukowitz \& Williams (2002). 94

Figura 9 - Modelo dos processos essenciais da gestão do conhecimento, baseado em Probst et Al. (2002).

Figura 10 - Causas de perturbação em processos de M\&A (Harding \& Rovit, 2005).

Figura 11 - Fatores de sucesso em processos de M\&A (Harding \& Rovit, 2005). 135

Figura 12 - Arcabouço do Generic Integrative Model de MacedoSoares (2000). 145

Figura 13 - Diagrama referencial de variáveis e suas relações, com base no Modelo GI de Macedo Soares (2000).

Figura 14 - Diagrama referencial de variáveis e suas relações, com base no modelo Gl de Macedo-Soares (2000) com inclusão da variável KM, segundo o modelo de Probst et Al. (2002).

Figura 15 - Diagrama das etapas do processo de pesquisa, com base em Cooper \& Schindler (2004:69/367).

Figura 16 - Evolução do faturamento em milhares de reais da empresa MHW, em termos nominais.

Figura 17 - Quadro dos fatores macroambientais implicados no desempenho da estratégia da empresa Xerox|MHW.

Figura 18 - Quadro dos atores estratégicos implicados no desempenho da estratégia da empresa Xerox|MHW. 
Figura 19 - Diagrama de variáveis e relações do processo de M\&A envolvendo a USB Xerox e a MHW.

Figura 20 - Diagrama evidenciando o efeito da variável processos essenciais para efetivação da KM.

Figura 21 - Diagrama evidenciando a influência da variável KM sobre o desempenho da estratégia.

Figura 22 - Diagrama de variáveis e relações evidenciando a relação da variável integração organizacional com a KM e o desempenho da estratégia.

Figura 23 - Diagrama de variáveis e relações evidenciando a relação da variável integração cultural com a KM e o desempenho da estratégia.

Figura 24 - Diagrama de variáveis e relações evidenciando a relação da variável sinergias de custo e receita com a KM e o desempenho da estratégia.

Figura 25 - Cenários de possíveis relações entre fatores organizacionais, inclusive a $\mathrm{KM}$, e macroambientais, e suas implicações relativamente ao sucesso do desempenho da estratégia organizacional. 


\section{Lista de Quadros}

Quadro I - Atributos referenciais necessários / desejáveis das variáveis principais independentes, (fatores organizacionais) relativos a empresas de tecnologia em mercados emergentes, como o de elearning, em caso de adoção da gestão do conhecimento (KM) como elemento essencial da estratégia competitiva de liderança no mercado por diferenciação orientada ao cliente.

Quadro II - Indicadores referenciais de desempenho da estratégia (variável principal dependente), relativos a empresas de tecnologia em mercados emergentes, como o de e-learning, em caso de adoção da gestão do conhecimento (KM) como elemento essencial da estratégia competitiva de liderança no mercado por diferenciação orientada ao cliente.

Quadro III - Caracterização e observações, com base nos resultados da pesquisa, dos atributos e indicadores das variáveis do fenômeno, segundo categorias. 


\section{Lista de Tabelas}

Tabela I - Receitas em cinco anos. 180

Tabela II - Preço das ações em cada ano. 180

Tabela III - Posição no rank das 500 maiores da revista Fortune. 180 
"Nos últimos 50 anos, as empresas japonesas produziram em um ambiente em que a única certeza era a incerteza. [...] A competição era uma batalha constante e penosa [...]. Em uma análise retrospectiva, isso foi uma felicidade, uma vez que não adquiriram os ônus habituais do sucesso - inclusive a complacência e a arrogância [...]".

(Criação de conhecimento na empresa, Nonaka \& Takeuchi, 1997:2/3) 